\title{
Full genetic analysis for genome-wide association study of Fangji: a powerful approach for effectively dissecting the molecular architecture of personalized traditional Chinese medicine
}

\author{
Gang $\mathrm{CHEN}^{1,2, *}$, Wen-da XUE ${ }^{1,2}$, Jun $\mathrm{ZHU}^{3}$ \\ ${ }^{1}$ Center for Translational Systems Biology and Neuroscience and Key Laboratory of Integrative Biomedicine for Brain Diseases, Nanjing \\ University of Chinese Medicine, Nanjing 210023, China; ${ }^{2}$ Co-innovation Center of Neuroregeneration, Nantong University, Nantong \\ 226001, China; ${ }^{3}$ Institute of Bioinformatics, Zhejiang University, Hangzhou 310058, China
}

\begin{abstract}
Elucidation of the systems biology foundation underlying the effect of Fangji, which are multi-herbal traditional Chinese medicine (TCM) formulas, is one of the major aims in the field. The numerous bioactive ingredients of a Fangji deal with the multiple targets of a complex disease, which is influenced by a number of genes and their interactions with the environment. Genome-wide association study (GWAS) is an unbiased approach for dissecting the genetic variants underlying complex diseases and individual response to a given treatment. GWAS has great potential for the study of systems biology from the point of view of genomics, but the capacity using current analysis models is largely handicapped, as evidenced by missing heritability. Recent development of a full genetic model, in which gene-gene interactions (dominance and epistasis) and gene-environment interactions are all considered, has addressed these problems. This approach has been demonstrated to substantially increase model power, remarkably improving the detection of association of GWAS and the construction of the molecular architecture. This analysis does not require a very large sample size, which is often difficult to meet for a GWAS of treatment response. Furthermore, this analysis can integrate other omic information and allow for variations of Fangji, which is very promising for Fangjiomic study and detection of the sophisticated molecular architecture of the function of Fangji, as well as for the delineation of the systems biology of personalized medicine in TCM in an unbiased and comprehensive manner.
\end{abstract}

Keywords: traditional Chinese medicine; Fangji; GWAS; full genetic model; gene-gene interaction; personalized medicine

Acta Pharmacologica Sinica (2018) 39: 906-911; doi: 10.1038/aps.2017.137; published online 8 Feb 2018

\section{Introduction}

One of the biggest challenges in current medicine is the difficulty in preventing and treating a large number of chronic and complex diseases, such as diabetes, cardiovascular and cerebrovascular diseases, cancer, and Alzheimer's disease $^{[1]}$. It has been widely demonstrated that the etiology of complex diseases is the result of a combination of multiple environmental and genetic factors ${ }^{[2]}$; thus, limited clinical outcomes may arise from intervention with one target among the complicated network of the disease. The need to apply multi-target combination therapeutic strategy has been increasingly recognized ${ }^{[3]}$. On the other hand, with the

\footnotetext{
*To whom correspondence should be addressed.

E-mail hdn_2001@yahoo.com

Received 2017-07-09 Accepted 2017-08-29
}

advance of genomics technology, precision medicine in recent years has aimed to achieve personalized treatment based on each individual's genetic disposition, which circumscribes the differing response to treatment ${ }^{[4]}$. Interestingly, traditional Chinese medicine (TCM) systematically examines the symptoms of the disease throughout the whole body, identifies the patterns or models of disease syndromes (Zheng), and prescribes a corresponding Fangji consisting of multiple herbs to individually treat patients. Therefore, Fangji are not only multi-targeted but are also used for personalized medicine, which has unique research and clinical value. The advances in high-throughput genomic and other omics technology provide powerful tools for investigating the systems biology of the multi-targeted and personalized nature of Fangji ${ }^{[5]}$.

Complex diseases, as complex or quantitative traits, are determined by many genes, most of which have small effects, 
and their interactions or interaction with the environment ${ }^{[6]}$. The contributing genes are called quantitative trait genes. The tremendously high diversity of genetic variation and its interaction with the environment contributes to the difference in pathogenesis and development of diseases ${ }^{[7]}$ and lays the foundation for the variety of patterns of TCM syndromes (or Zheng) of the disease, as well as the corresponding personalized treatment. Fangji typically consist of a number of herbs, according to the principles of Monarch, Minister, Assistant and Guide (Jun, Chen, Zuo and Shi) and other characteristics of compatibility. Each constituent herb usually contains hundreds or thousands of different compounds ${ }^{[8]}$. The overall efficacy is only infrequently accounted for by one or a small number of compounds. Rather, it is a synergy of various lowconcentration, small-effect compounds, together with few enriched and active compounds. A Fangji, or its many smalleffect active compounds, acts on the signaling systems controlled by many small-effect genes, modifying the molecular architecture susceptible to diseases, which may underlie the basis of the systems biology of personalized treatment with Fangji. Therefore, we can infer the particular molecular architecture that mediates the personalized medicine of Fangji by dissecting the genetic variants influencing the likelihood of response to Fangji, which can be implemented with genomewide association studies (GWAS) and/or integrated with other omic approaches.

\section{GWAS and full genetic model}

\section{The success and problems of GWAS}

Based on the completion of human genome sequencing and the development of high-throughput genotyping techniques, GWAS analysis of complex diseases at the genome level comprehensively reveals the genetic factors and the network for pathogenesis and development of disease and drug response ${ }^{[9]}$. In a typical GWAS, each individual's whole genome is analyzed by using millions of single nucleotide polymorphisms (SNPs) as molecular genetic markers, and the genetic variants are associated with variations in the complex traits of interest in the population ${ }^{[10]}$. Human complex traits include physiological traits such as height and body weight, as well as disease traits such as hypertension and glaucoma, which are determined by a variety of genes, their environment, and their interactions ${ }^{[11-13]}$. Complex diseases, TCM Zheng, and response to drugs are all complex traits and can be studied using the GWAS approach. In 2005, the first important GWAS study reported age-related retinal macular degeneration ${ }^{[14]}$. Since then, findings from GWAS research have rapidly increased for different diseases, including cancer, diabetes, autism, systemic lupus erythematosus, psoriasis and response to drugs ${ }^{[15,16]}$. As of June 2017, nearly 3000 studies reported approximately 37000 associations with 31500 SNPs (http://www.ebi.ac.uk/ gwas/home). As with other phenotypes, GWAS can directly associate different TCM phenotypes such as Zheng, constituent, or response to Fangji with genome-wide genetic variability and are powerful for resolving the molecular basis of TCMrelated phenotypes. This analysis represents an unbiased investigation of the molecular substrates associated with the phenotype and thus does not require any assumptions about the mechanism, anatomy and physiology of the traits studied, which is particularly important and useful for TCM-related phenotypic studies because many of them are not clear.

Over the last few decades, GWAS analysis has revealed several important characteristics. The vast majority of associations were not located in the coding region of the functional protein, implicating that most of the associated variants were in regulatory loci ${ }^{[17,18]}$; additionally, each associated variant makes only a very small contribution to the disease phenotype ${ }^{[19,20]}$. These led to the conclusion that it is not the variants of the functional or core genes but the large number of lowcontribution regulatory or peripheral genes that constitute the genetic network for complex disease $\mathrm{e}^{[21]}$. This conclusion may need to be addressed for the simplistic precision medicine model, in which only one or a few number of signaling pathways are considered. Although GWAS has achieved great success, current GWAS still have significant limitations. One of the major limitations is so-called missing heritability ${ }^{[22-24]}$. GWAS has been expected to detect a large amount of association, as high-resolution genetic markers have been used in GWAS. However, for most disease phenotypes, the total heritability from association discovered by GWAS is very low, and thus the majority of associations cannot be detected ${ }^{[25,26]}$. For example, based on pedigree studies, human height is $80 \%$ genetically determined. Up to now, approximately 50 SNPs associated with human height have been detected, together accounting for only $5 \%$ of height variation. This result can be partly ascribed to disease heterogeneity, rare variation, epigenetics, etc ${ }^{[27]}$. An important reason, however, is that the current model for association analysis is insufficient.

\section{The full genetic model of GWAS}

According to the principle of genetics, the gene effect consists of an additive effect, a dominance effect and an epistatic effect. For the additive effect, each gene acts independently. For the dominance and epistatic effects, there is genetic interaction between alleles and between non-alleles, respectively. However, the existing GWAS analysis essentially analyzes only additive effects, failing to discover gene-gene and geneenvironment interactions ${ }^{[28,29]}$. As these interactions play important roles in determining complex traits, inadequate consideration of these interactions by the current GWAS analysis significantly underestimates associations ${ }^{[30-32]}$. To address this, a novel mixed-liner GWAS analysis model considering various interactions has been developed and implemented $^{[33]}$. This model has been applied for re-analysis of some GWAS data for complex traits, including human alcohol dependence, cholesterol level, body mass index, coronary heart disease, mouse anxiety-like behavior, crops, etc ${ }^{[33-36]}$. The results have demonstrated that, in addition to replicating associations reported previously, an appreciable number of new associations have also been detected, particularly in dominance or epistatic mode. Subsequently, the molecular architecture of those associated genes detected 
has been constructed. For example, alcoholism, or alcohol dependence, is a complex disease that is approximately $50 \%$ genetically determined ${ }^{[37,38]}$. However, until now, even though dozens of GWAS on alcoholism have been performed, only low heritability has been discovered using the conventional GWAS, less than $1 \%{ }^{[39]}$. Chen et al ${ }^{[29]}$ reanalyzed the dataset from 3838 subjects to discover quantitative traits of alcohol dependence symptom count (ADSC), considering additive, dominance, and epistatic effects and their interactions with the environment. This reanalysis detected 20 quantitative trait SNPs associated with ADSC. Five associations have been previously reported from different studies. Additionally, the analysis revealed that the replicated association with the gene ADH1C was highly significant in a dominance inheritance mode and was predicted to increase the risk of ADSC at a considerably high level. Interestingly, an environmental factor, co-morbidity of substance dependence, also influenced the impact of the ADH1C variant on ADSC, dependent on the type of the substance dependence: only co-morbid opiate or marijuana dependence, but not nicotine or cocaine, showed the effect, indicating the complexity of the geneenvironment interaction. Fifteen new associations have also been identified in variants, including four novel genes, two non-coding RNA and two epistasis loci. Two SNPs interacted in an additive $\times$ additive or additive $\times$ dominance manner, with one within a gene, $P T P R G$, encoding a protein tyrosine phosphatase (PTP), the other one near a gene, ANGPT1, encoding a PTP receptor, supporting the validity of the model for estimation of the epistatic effect. Both ADH1C and ANGPT1 have been found to be significantly or nominally associated with alcohol dependence ${ }^{[40-42]}$, and other family members of PTP are associated with the disorder ${ }^{[43]}$. From the results of the reanalysis, over 20 percent of total heritability was detected, much closer to the results from family and twin studies; dominance and epistatic effects accounted for over 50 percent of the total estimated heritability. In contrast, in the first paper on GWAS using conventional analysis of the same dataset, no association reached genome-wide significant level ${ }^{[44]}$. To make the comparison more straightforward, GWAS of human total cholesterol level was carried out using a conventional single locus additive approach, including PLINK analysis and GCTA analysis. Only two and one significant association, respectively, reached genome-wide association level. Use of the full model, however, discovered 15 significant associations, and the dominance and epistatic effects accounted for approximately $60 \%$ of total heritability. Finally, simulations analysis further supported the validity of the full model ${ }^{[35]}$. In sum, the full genetic model has been demonstrated to improve the unbiased detection of a significant number of GWAS associations and has remarkably resolved the problem of missing heritability.

The full model can analyze not only the association from the sole dataset of genome but also from integrated data of multiple omics, including genome, transcriptome, proteome, metabolome, etc, to systematically identify the biological information flow of specific molecular architecture that influences the phenotype of interest ${ }^{[33]}$. Therefore, use of the full genetic model makes it plausible to comprehensively and systematically dissect how molecular structure, influenced by numerous small-effect genetic variability, is disturbed by the action of the environment or stress to govern the eventual occurrence of variations in the physiological phenotype, disease, TCM syndromes and drug response, thus offering comparatively high practical value in different fields, including Fangji study (Figure 1).

\section{Perspective on the application of full genetic model GWAS to Fangjiomics}

Identification of genetic factors underlying individual difference in response to drug treatment is key for precision medicine $^{[45,46]}$. Paradoxically, GWAS of drug response, including adverse reactions to a drug, composes less than $10 \%$ of the total number of published GWAS reports and has achieved genome-wide significant association with less than 300 SNPs, averaging 2 or less per study ${ }^{[47,48]}$. Despite this limitation, these studies have still provided valuable information on the mechanisms underlying drug distribution, efficacy and toxicology ${ }^{[49-51]}$. For example, hepatic CYP2C19 enzyme, a CYP450 superfamily member, regulates the metabolism of many drugs, including antidepressants ${ }^{[22,53]}$, and their genetic variants were detected to have a significant effect on the choice of appropriate types and doses of clinical anticoagulants. Studies have also revealed the marked effects of ethnicity on drug response and toxicology ${ }^{[54-56]}$.

To reveal the mechanisms and factors that influence the effects of Fangji, GWAS is needed for unbiased identification of the complicated underlying molecular architecture. Currently, no GWAS regarding Fangji have been reported. Even for GWAS of conventional drug response, the number of the studies is still relatively limited, partly due to the requirement of the large sample size for detection of a considerable number of associations using the conventional GWAS approach, which is more difficult for drug response studies ${ }^{[48]}$. As mentioned above, this obstacle may be at least partly overcome by using full genetic model analysis, by which an appreciable number of significant associations can be disclosed so that molecular architecture for Fangji's action can be constructed without a very large sample size ${ }^{[29]}$. Based on the full genetic model GWAS analysis, it is possible to reveal the systems biology governing the distribution and pharmacodynamics of Fangji, as well as the associated genes and network of efficacy or side effects of Fangij ${ }^{[33]}$. Additionally, the full genetic model also offers more flexibility by allowing certain variations in Fangji in the model. This is very important, as in the clinical practice of TCM, there are constant changes in the composition of a given Fangji, called addition and subtraction, as well as changes in doses or frequency to individually treat the patients. In the full genetic model analysis, these changes in Fangji can all be used as covariates to reveal their involvement in the molecular and phenotypical impacts of Fangji. Furthermore, the full genetic model is also capable of dissecting the molecular architecture of the therapeutic 
or adverse effects of variable constituents or compounds of Fangji, or Fangjiomics, by using high-efficient phytochemical isolation and identification technology (Figure 1).

In conclusion, understanding the genetic networks and molecular architecture of Fangji remains a main challenge in the field. Recent progress in GWAS has proven very successful for unbiased dissection of new genetic underpinnings of different diseases, physiological traits and drug responses, although marked limitations in missing heritability have handicapped the capability to detect associations, partly due to the inability to capture gene-gene and gene-environment interactions using a simple additive model analysis. The development of a full genetic model considering influences from all genetic and gene-environment interactions has been very successful and substantially reduced the missing heritability evidenced by the conventional approach. It has been held that the action of Fangii is a result of synergy of many small-effect ingredients or compounds, in a way similar to a quantitative trait contributed by numerous small- effect genetic factors. It will thus be intriguing to clarify the molecular and genetic machinery underlying the mechanisms of Fangii and the phytochemical substrates of Fangjiomics. To this end, full genetic model analysis of GWAS from human studies or animal models is instrumental. GWAS, or GWAS combined with analysis of other omics, will offer novel insights with a systematic and dynamic bioinformatic flow regarding Fangji's mechanism.

\section{References}

1 Kilmer G, Roberts H, Hughes E, Li Y, Valluru B, Fan A, et al. Surveillance of certain health behaviors and conditions among states and selected local areas - Behavioral Risk Factor Surveillance System (BRFSS), United States, 2006. MMWR Surveill Summ 2008; 57: 1-188.

2 Stender S, Kozlitina J, Nordestgaard BG, Tybjaerg-Hansen A, Hobbs $\mathrm{HH}$, Cohen JC. Adiposity amplifies the genetic risk of fatty liver disease conferred by multiple loci. Nat Genet 2017; 49: 842-7.

3 Bawa P, Pradeep P, Kumar P, Choonara YE, Modi G, Pillay V. Multitarget therapeutics for neuropsychiatric and neurodegenerative

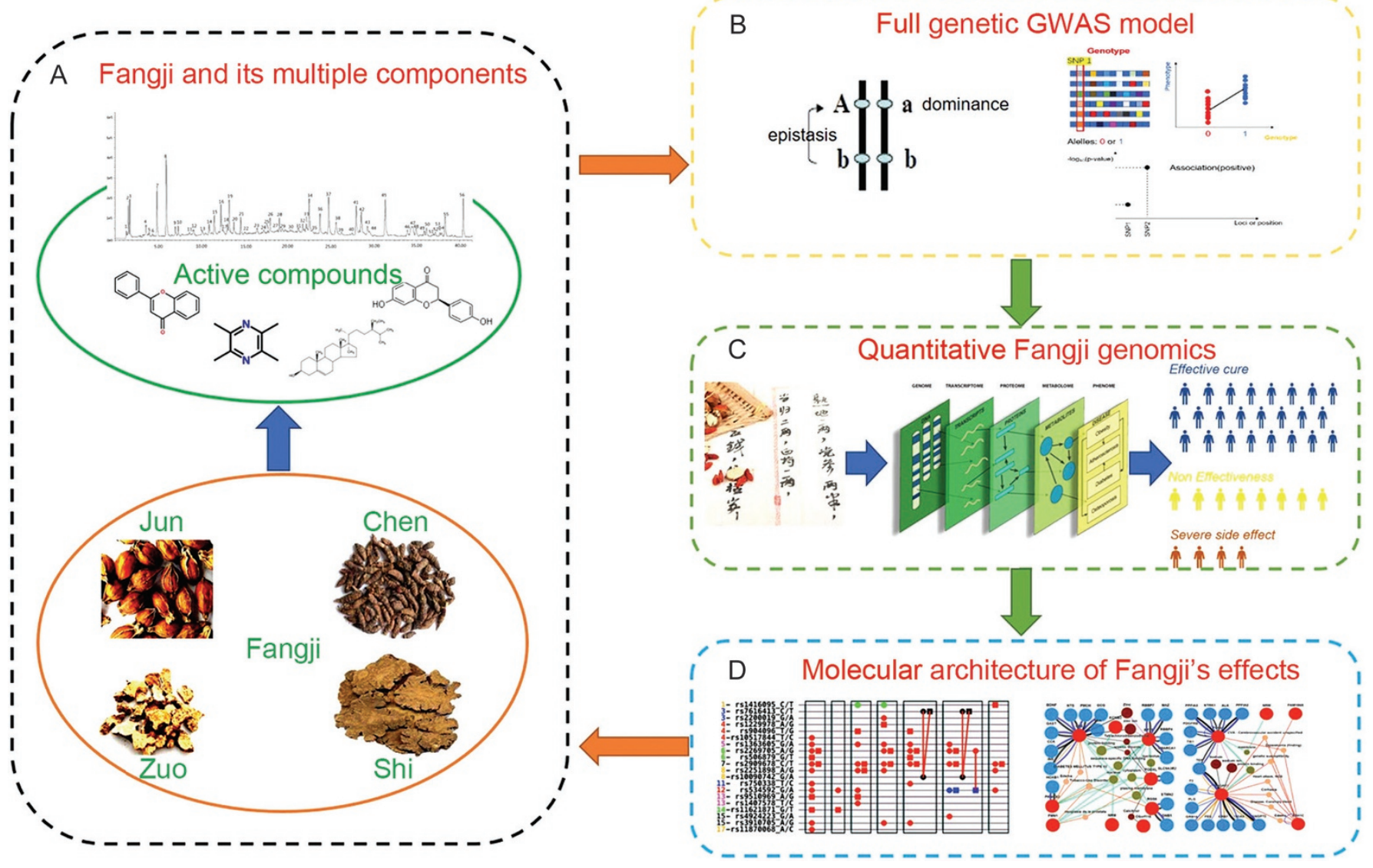

Figure 1. Dissection of the molecular architecture of Fangjiomics using the full genetic analysis of genome-wide association study. (A) The multiherbal Fangji and the rule of compatibility. Each herb consists of thousands of compounds, some of which have very small effects by themselves and collectively treat the disease. (B) The molecular architecture for individual difference in response to Fangji can be examined using the GWAS approach with full genetic analysis, in which dominance and epistatic (genetic interaction between alleles and non-alleles, respectively) effects are included. (C) The full genetic model analysis of GWAS is able to identify the efficacy or side effects of Fangii, with the variations of doses or herbal constituents considered. The analysis can also integrate other omic information, such as transcriptome or proteome, powerfully deciphering the systems biology of the personalized medicine of Traditional Chinese Medicine using Fangji. (D) The analysis can detect a large number of associated SNPs and genes, and the molecular architecture underlying Fangji's effect can be constructed. 
disorders. Drug Discov Today 2016; 21: 1886-914.

4 Carrasco-Ramiro F, Peiro-Pastor R, Aguado B. Human genomics projects and precision medicine. Gene Ther 2017; 24: 551-61.

5 Duan DD, Wang Z, Zhang BL, Wang YY. Fangjiomics: revealing adaptive omics pharmacological mechanisms of the myriad combination therapies to achieve personalized medicine. Acta Pharmacol Sin 2015; 36: 651-3.

6 Meyer NJ, Calfee CS. Novel translational approaches to the search for precision therapies for acute respiratory distress syndrome. Lancet Respir Med 2017; 5: 512-23.

7 Riordan JD, Nadeau JH. From peas to disease: modifier genes, network resilience, and the genetics of health. Am J Hum Genet 2017; 101: 177-91.

8 Zhang JH, Zhu Y, Fan XH, Zhang BL. Efficacy-oriented compatibility for component-based Chinese medicine. Acta Pharmacol Sin 2015; 36 : 654-8.

9 Ainsworth HF, Shin SY, Cordell HJ. A comparison of methods for inferring causal relationships between genotype and phenotype using additional biological measurements. Genet Epidemiol 2017; 41: 577-86.

10 Lu YF, Goldstein DB, Angrist M, Cavalleri G. Personalized medicine and human genetic diversity. Cold Spring Harb Perspect Med 2014; 4: a008581.

11 Darabos C, Moore JH. Genome-wide epistasis and pleiotropy characterized by the bipartite human phenotype network. Methods Mol Biol 2015; 1253: 269-83.

12 Ge T, Chen CY, Neale BM, Sabuncu MR, Smoller JW. Phenomewide heritability analysis of the UK Biobank. PLoS Genet 2017; 13: e1006711.

13 Liu C, Kraja AT, Smith JA, Brody JA, Franceschini N, Bis JC, et al. Meta-analysis identifies common and rare variants influencing blood pressure and overlapping with metabolic trait loci. Nat Genet 2016; 48: 1162-70.

14 Haines JL, Hauser MA, Schmidt S, Scott WK, Olson LM, Gallins P, et al. Complement factor $\mathrm{H}$ variant increases the risk of age-related macular degeneration. Science 2005; 308: 419-21.

15 Mackey DA, Hewitt AW. Genome-wide association study success in ophthalmology. Curr Opin Ophthalmol 2014; 25: 386-93.

16 Zhang XJ, Huang W, Yang S, Sun LD, Zhang FY, Zhu QX, et al. Psoriasis genome-wide association study identifies susceptibility variants within LCE gene cluster at 1q21. Nat Genet 2009; 41: 205-10.

17 Marouli E, Graff M, Medina-Gomez C, Lo KS, Wood AR, Kjaer TR, et al. Rare and low-frequency coding variants alter human adult height. Nature 2017; 542: 186-90.

18 Yao S, Guo Y, Dong SS, Hao RH, Chen XF, Chen YX, et al. Regulatory element-based prediction identifies new susceptibility regulatory variants for osteoporosis. Hum Genet 2017; 136: 963-74.

19 Heath AC, Whitfield JB, Martin NG, Pergadia ML, Goate AM, Lind PA, et al. A quantitative-trait genome-wide association study of alcoholism risk in the community: findings and implications. Biol Psychiatry 2011; 70: 513-8.

20 Henriksen MG, Nordgaard J, Jansson LB. Genetics of schizophrenia: overview of methods, findings and limitations. Front Hum Neurosci 2017; 11: 322.

21 Boyle EA, Li YI, Pritchard JK. An expanded view of complex traits: from polygenic to omnigenic. Cell 2017; 169: 1177-86.

22 Manolio TA, Collins FS, Cox NJ, Goldstein DB, Hindorff LA, Hunter DJ, et al. Finding the missing heritability of complex diseases. Nature 2009; 461: 747-53.

23 Nolte IM, van der Most PJ, Alizadeh BZ, de Bakker PI, Boezen HM, Bruinenberg $\mathrm{M}$, et al. Missing heritability: is the gap closing? An analysis of 32 complex traits in the Lifelines Cohort Study. Eur J Hum Genet 2017; 25: 877-85.

24 Vineis P, Pearce N. Missing heritability in genome-wide association study research. Nat Rev Genet 2010; 11: 589.

25 Demirkan A, Amin N, Isaacs A, Jarvelin MR, Whitfield JB, Wichmann $\mathrm{HE}$, et al. Genetic architecture of circulating lipid levels. Eur J Hum Genet 2011; 19: 813-9.

26 Naukkarinen J, Surakka I, Pietilainen KH, Rissanen A, Salomaa V, Ripatti S, et al. Use of genome-wide expression data to mine the "Gray Zone" of GWA studies leads to novel candidate obesity genes. PLoS Genet 2010; 6: e1000976.

27 Zuk O, Hechter E, Sunyaev SR, Lander ES. The mystery of missing heritability: Genetic interactions create phantom heritability. Proc Natl Acad Sci U S A 2012; 109: 1193-8.

28 Tohkin M, Kaniwa N, Saito Y, Sugiyama E, Kurose K, Nishikawa J, et al. A whole-genome association study of major determinants for allopurinol-related Stevens-Johnson syndrome and toxic epidermal necrolysis in Japanese patients. Pharmacogenomics J 2013; 13: 60-9.

29 Chen G, Zhang F, Xue W, Wu R, Xu H, Wang K, et al. An association study revealed substantial effects of dominance, epistasis and substance dependence co-morbidity on alcohol dependence symptom count. Addict Biol 2016; 22: 1475-85.

30 Chu M, Zhang R, Zhao Y, Wu C, Guo H, Zhou B, et al. A genome-wide gene-gene interaction analysis identifies an epistatic gene pair for lung cancer susceptibility in Han Chinese. Carcinogenesis 2014; 35: 572-7.

31 Meng Y, Groth S, Quinn JR, Bisognano J, Wu TT. An exploration of gene-gene interactions and their effects on hypertension. Int J Genomics 2017; 2017: 7208318.

32 Verma SS, Cooke Bailey JN, Lucas A, Bradford Y, Linneman JG, Hauser $\mathrm{MA}$, et al. Epistatic gene-based interaction analyses for glaucoma in eMERGE and NEIGHBOR consortium. PLoS Genet 2016; 12: e1006186.

33 Zhang FT, Zhu ZH, Tong XR, Zhu ZX, Qi T, Zhu J. Mixed linear model approaches of association mapping for complex traits based on Omics variants. Sci Rep 2015; 5: 10298.

34 Luo X, Ding Y, Zhang L, Yue Y, Snyder JH, Ma C, et al. Genomic prediction of genotypic effects with epistasis and environment Interactions for yield-related traits of rapeseed (Brassica napus L). Front Genet 2017; 8: 15.

35 Monir MM, Zhu J. Comparing GWAS results of complex traits using full genetic model and additive models for revealing genetic architecture. Sci Rep 2017; 7: 38600.

36 Zhu J, Loos RJ, Lu L, Zong G, Gan W, Ye X, et al. Associations of genetic risk score with obesity and related traits and the modifying effect of physical activity in a Chinese Han population. PLoS One 2014; 9: e91442.

37 Kendler KS, Heath AC, Neale MC, Kessler RC, Eaves $\sqcup$. A populationbased twin study of alcoholism in women. JAMA 1992; 268: 187782.

38 Prescott CA, Kendler KS. Genetic and environmental contributions to alcohol abuse and dependence in a population-based sample of male twins. Am J Psychiatry 1999; 156: 34-40.

39 Palmer RH, McGeary JE, Francazio S, Raphael BJ, Lander AD, Heath $\mathrm{AC}$, et al. The genetics of alcohol dependence: advancing towards systems-based approaches. Drug Alcohol Depend 2012; 125: 17991.

40 Chen YC, Lu RB, Peng GS, Wang MF, Wang HK, Ko HC, et al. Alcohol metabolism and cardiovascular response in an alcoholic patient homozygous for the ALDH2*2 variant gene allele. Alcohol Clin Exp 
Res 1999; 23: 1853-60.

41 Gelernter J, Kranzler HR, Sherva R, Almasy L, Koesterer R, Smith $\mathrm{AH}$, et al. Genome-wide association study of alcohol dependence: significant findings in African- and European-Americans including novel risk loci. Mol Psychiatry 2014; 19: 41-9.

42 Biernacka JM, Geske JR, Schneekloth TD, Frye MA, Cunningham JM, Choi DS, et al. Replication of genome wide association studies of alcohol dependence: support for association with variation in ADH1C. PLoS One 2013; 8: e58798.

43 Zuo L, Zhang CK, Wang F, Li CS, Zhao H, Lu L, et al. A novel, functional and replicable risk gene region for alcohol dependence identified by genome-wide association study. PLoS One 2011; 6: e26726.

44 Bierut L, Agrawal A, Bucholz KK, Doheny KF, Laurie C, Pugh E, et al. A genome-wide association study of alcohol dependence. Proc Natl Acad Sci U S A 2010; 107: 5082-7.

45 Uzilov AV, Ding W, Fink MY, Antipin Y, Brohl AS, Davis C, et al. Development and clinical application of an integrative genomic approach to personalized cancer therapy. Genome Med 2016; 8: 62.

46 Perez V, Salavert A, Espadaler J, Tuson M, Saiz-Ruiz J, Saez-Navarro $C$, et al. Efficacy of prospective pharmacogenetic testing in the treatment of major depressive disorder: results of a randomized, double-blind clinical trial. BMC Psychiatry 2017; 17: 250.

47 Cardon LR, Harris T. Precision medicine, genomics and drug discovery. Hum Mol Genet 2016; 25: R166-R172.

48 Giacomini KM, Yee SW, Mushiroda T, Weinshilboum RM, Ratain MJ, Kubo M. Genome-wide association studies of drug response and toxicity: an opportunity for genome medicine. Nat Rev Drug Discov
2017; 16: 1.

49 Sriretnakumar V, Huang E, Muller DJ. Pharmacogenetics of clozapine treatment response and side-effects in schizophrenia: an update. Expert Opin Drug Metab Toxicol 2015; 11: 1709-31.

50 Maronas O, Latorre A, Dopazo J, Pirmohamed M, Rodriguez-Antona C, Siest G, et al. Progress in pharmacogenetics: consortiums and new strategies. Drug Metab Pers Ther 2016; 31: 17-23.

51 Dubovsky SL. The usefulness of genotyping cytochrome P450 enzymes in the treatment of depression. Expert Opin Drug Metab Toxicol 2015; 11: 369-79.

52 Backman JD, O'Connell JR, Tanner K, Peer CJ, Figg WD, Spencer $\mathrm{SD}$, et al. Genome-wide analysis of clopidogrel active metabolite levels identifies novel variants that influence antiplatelet response. Pharmacogenet Genomics 2017; 27: 159-63.

53 Hamilton SP. The promise of psychiatric pharmacogenomics. Biol Psychiatry 2015; 77: 29-35.

54 Nicoletti P, Aithal GP, Bjornsson ES, Andrade RJ, Sawle A, Arrese M, et al. Association of liver injury from specific drugs, or groups of drugs, with polymorphisms in HLA and other genes in a genome-wide association study. Gastroenterology 2017; 152: 1078-89.

55 Yang $X$, Zhang B, Zhu J. Functional genomics- and networkdriven systems biology approaches for pharmacogenomics and toxicogenomics. Curr Drug Metab 2012; 13: 952-67.

56 Gong Y, McDonough CW, Beitelshees AL, El Rouby N, Hiltunen TP, O'Connell JR, et al. PTPRD gene associated with blood pressure response to atenolol and resistant hypertension. J Hypertens 2015; 33: $2278-85$. 